\title{
Mapa geológico y base de datos geocronológicos de Sonora, México ${ }^{\text {尔 }}$
}

\section{Geologic map and geochronology data base of Sonora, Mexico}

\author{
Carlos M. González-León*a , Gilberto S. Moreno-Hurtado ${ }^{\mathrm{b}}$ \\ ${ }^{a}$ Estación Regional del Noroeste, Instituto de Geología, Universidad Nacional Autónoma de México, L. D. Colosio S/N Madrid, Hermosillo, México, 83000 \\ ${ }^{b}$ Ingeniería en Geociencias, Universidad Estatal de Sonora, Unidad Académica Hermosillo, Ley Federal del Trabajo s/n, Col. Apolo, Hermosillo, México, 83100
}

\section{Resumen}

Este trabajo presenta un mapa geológico del Estado de Sonora, México, que fue compilado a la escala 1:1,000,000, y está acompañado de una base de datos isotópicos. Ambos se elaboraron a partir de la compilación de trabajos publicados y se incluyen observaciones e interpretaciones propias. El trabajo es la actualización de una versión publicada en 2006 que fue elaborada sobre mapas topográficos base y se digitalizó generando nuevos datos vectoriales, adicionándole el mapa de relieve. El mapa se forma por 33 unidades cartográficas reconocidas por sus litologías y edades, y muestra 1,129 edades $\mathrm{U}-\mathrm{Pb}, \mathrm{K}-\mathrm{Ar}$ y ${ }^{40} \mathrm{Ar} /{ }^{39} \mathrm{Ar}$ de rocas ígneas y de mineralización en Re-Os.

Palabras clave: Mapa geológico digital; base de datos isotópicos; Estado de Sonora; México

\begin{abstract}
This work presents a geologic map of the state of Sonora, Mexico, that was compiled to the scale of 1:1,000,000 and it is accompanied by an isotopic database. Both are compilations of published works and include personal observations and interpretations. This is an updated version of a map published in 2006 which was elaborated on topographic base maps and is now re-digitized adding a relief map. The map is composed of 33 cartographic units, each recognized on the basis of their lithology and age and shows the location of $1,129 \mathrm{U}-\mathrm{Pb}, \mathrm{K}-\mathrm{Ar}$, and ${ }^{40} \mathrm{Ar} /{ }^{39} \mathrm{Ar}$ isotopic ages of igneous rocks and Re-Os ages of mineralization.
\end{abstract}

Keywords: Digital geologic map; isotopic database; Sonora state; Mexico

\section{Introducción}

La cartografía geológica de Sonora ha sido motivo de interés desde finales del Siglo XIX. En 1888 y después de su viaje de reconocimiento a la región del sismo del 3 de mayo de 1887 en el noreste de Sonora, J. G. Aguilera publicó la cartografía a escala 1:1,000,000 que levantó entre Hermosillo y Agua Prieta (Aguilera, 1888; Suter, 2007). En 1939, R. E. King publicó la geología de la mitad sur del estado, donde reconoció varias unidades litológicas, a muchas de las cuales les asignó edades correctas con base en su contenido fosilífero (King, 1939).

\footnotetext{
(C) C. M. González-León, G. S. Moreno-Hurtado

This is an Open Access article distributed under the terms of the Creative Commons Attribution License (https://creativecommons.org/licenses/by-nc-sa/4.0/), which permits non-commencial sharing of the work and adaptions, provided the original work is properly cited and the new creations are licensed under identical terms.

*E-mail address: cmgleon@unam.mx
}

El primer mapa que reportó la geología de Sonora fue publicado por López-Ramos (1974), a escala 1:5,000,000 y se basó en trabajos cartográficos regionales que para ese tiempo se habían realizado, como el de 1969 del norte del estado por las Naciones Unidas, el de Salas (1968) y Merriam (1972), entre otros. Otros trabajos de cartografía y geocronología de la región costera y de la Isla Tiburón, fueron publicados por Gastil y Krummenacher (1976), Gastil y Krummenacher (1977) y Gastil et al. (1979). A principios de los 80's, la Dirección General de Geografía del Territorio Nacional publicó las cartas geológicas a escala 1:250,000, que fueron seguidas por su mapa a 1:1,000,000. Este último trabajo mostró una avanzada resolución geológica, distinguiendo la litoestratigrafía con una refinada asignación de edades. Posteriormente, la geología de Sonora quedó incluida en la Carta Geológica de la República Mexicana (Ortega-Gutiérrez et al., 1992), a la cual siguió la Carta Geológica de Sonora, 1:500,000, del Gobierno del Estado y el Centro de Estudios Superiores de Sonora (Fernández-Aguirre 
et al., 1993).

Una primera versión del trabajo aquí presentado fue una compilación de trabajos anteriores y fue publicado por la revista Digital Geosciences que dejó de estar activa unos años después (González-León et al., 2006). El Servicio Geológico Mexicano (SGM) publicó en el 2008 la carta Geológico-Minera del Estado de Sonora, a escala 1:500,000, la cual muestra gran refinamiento geológico, producto del importante avance que dicha dependencia ha tenido a partir de los 90's en la cobertura cartográfica-minera a escala 1:50,000. En ese mapa se reconocen las unidades litoestratigráficas de los diversos terrenos tectonoestratigráficos y está complementado con mapas de distribución de yacimientos, de edades paleontológicas e isotópicas.

El presente trabajo es una actualización del mapa geológico que fue publicado en la revista Digital Geosciences y tiene agregada una base de datos geocronológicos. Como sucede con la evolución del conocimiento, la información cartográfica presentada proviene de la compilación de trabajos publicados por diversos autores e instituciones, e inevitablemente, aunque en forma menor, contiene información e interpretaciones propias obtenidas en años de trabajo de campo en Sonora. Asimismo, la base de datos que lo acompaña es una compilación de edades publicadas por otros y por el primer autor y coautores.

\section{Métodos}

La compilación cartográfica original fue dibujada a mano sobre las cartas topográficas Tijuana y La Paz, publicadas por el INEGI (1990a y b), a escala 1:1,000,000, y posteriormente digitalizada en ArcGIS. La presente actualización se continuó sobre esa base digitalizada, modificando y generando nuevos datos vectoriales y agregando el mapa de relieve obtenido del Continuo de Elevaciones Mexicano (CEM), disponible en la página web del INEGI, https://www.inegi.org.mx/ app/geo2/elevacionesmex/ Para la actualización de la información se consultaron las cartas geológico-mineras, escala 1:50,000, que a la fecha han sido publicadas por el Servicio Geológico Mexicano y están disponibles en https://www . sgm.gob.mx/CartasDisponibles/, artículos científicos, guías de excursiones, reportes del USGS y tesis de estudiantes de diversas instituciones de educación superior. El material seleccionado fue georeferenciado y, en algunos casos, la posición de la traza de algunas fallas con clara expresión morfológica fue dibujada a partir del modelo de elevación. Por motivos de espacio, las referencias bibliográficas no incluyen a todos los autores que han contribuido al conocimiento de la geología del estado, y se incluyen solo las más representativas para el presente trabajo.

La compilación de datos isotópicos incluye edades que fueron obtenidas por los métodos de U-Pb, K-Ar y, ${ }^{40} \mathrm{Ar} /{ }^{39} \mathrm{Ar}$ y $\mathrm{Re} / \mathrm{Os}$, las cuales creemos que constituyen la mayoría de los datos reportados de Sonora, aunque algunos pudieron haber sido omitidos involuntariamente. Los datos fueron obtenidos de diferentes publicaciones, principalmente de artículos científicos y tesis, y algunos son datos no publicados. La información de cada muestra (clave, coordenadas, edad y error, método de datación, mineral, tipo de roca, localidad geográfica, autores y referencia bibliográfica) se ingresó a una hoja Excel (Material suplementario: Tabla 1) y constituye la tabla de atributos de la capa GEOCRONOLOGÍA del mapa interactivo que acompaña a esta publicación y puede consultarse desde el portal web de la revista. Para aquellas edades que no reportan coordenadas, se trató de inferir su ubicación con base en datos de referencia presentados en el trabajo. Durante el trabajo de compilación de edades y para controlar y visualizar su ubicación, estos datos se dispusieron en el servicio de QGIS Cloud.

\section{Unidades cartográficas}

La estructura geológica de Sonora está formada por la provincia Mazatzal en su parte norte (Nourse et al., 2005), por el bloque Caborca en su parte central (Campa y Coney, 1983) y por el terreno Cortés (Coney y Campa, 1987) en la parte sur. Cada uno de ellos tiene basamento diferente y están separados entre sí por fallas inferidas importantes. El Caborca se considera un fragmento de las provincias Mojave y/o Yavapai (Arvizu e Iriondo, 2015), que fue desplazado durante el Jurásico, desde el suroeste de los Estados Unidos hasta su posición actual, a lo largo de una hipotética falla de rumbo lateral izquierdo, que originalmente fue nombrada megacizalla Mojave-Sonora (Silver y Anderson, 1974). Sin embargo, otros autores han propuesto que dicho desplazamiento ocurrió en el Paleozoico tardío (Stone y Stevens, 1988; Dickinson y Lawton, 2001), o durante el Mesoproterozoico (Whitmeyer y Karlstrom, 2007), mientras que otros proponen la inexistencia de tal fallamiento (Poole et al., 2005; Molina-Garza e Iriondo, 2007). El terreno Cortés abarca la mitad sur de Sonora y se considera fue acrecionado al Caborca durante el Pérmico, siendo la sutura entre ambos el alóctono Sonora (Poole et al., 2005). En este mapa se ubican las trazas aproximadas del frente del alóctono Sonora (según Poole et al., 2005) y de la megacizalla Mojave-Sonora (según Anderson y Silver, 2005).

En este trabajo se reconocen 33 unidades cartografiables a escala 1:1,000,000. Algunas están restringidas a alguno de los terrenos antes mencionados y otras los traslapan. Varias de ellas son unidades reconocidas, como los grupos Barranca, Bisbee y Cabullona, o las formaciones Sierra de Santa Rosa, Tarahumara y Cocóspera. Las rocas volcánicas del Cretácico Superior y más jóvenes tienen distribución regional amplia, mientras que las del Cretácico Inferior y más antiguas presentan afloramientos restringidos, en parte al estar cubiertas por las primeras. Aunque el fallamiento extensional de rumbo, casi N-S, que empezó a partir del Oligoceno es profuso, éste favoreció que las rocas más antiguas fueran expuestas en los levantamientos asociados. Este fallamiento denominado de Sierras y Valles paralelos (Stewart y Roldán-Quintana, 1994) también determinó que casi la mitad del estado corresponda a grabens de dicho evento en los cuales se acumuló la Formación Báucarit y sedimentos y volcanismo de edad Plio-Cuaternaria. 
Las rocas más antiguas son de edad Paleoproterozoica y forman afloramientos pequeños y aislados en el bloque Caborca. Constituyen el Complejo Metamórfico Bámori (PEm) (Longoria et al., 1978), formado por paragneises y esquistos que están intrusionados por la unidad $\boldsymbol{P E} \boldsymbol{g}$, que incluye cuerpos graníticos a máficos metamorfizados con edades de ca. 1.8 a $1.6 \mathrm{Ga}$ y a granitoides Mesoproterozoicos con edades de ca. 1.4 y 1.1 Ga. El basamento Mazatzal $(\boldsymbol{P E})$, lo forma el Esquisto Pinal que tiene edades de depósito de ca. 1.6 Ga y está también cortado por los granitos Mesoproterozoicos.

La cubierta sedimentaria Neoproterozoica $(\boldsymbol{P E}-\boldsymbol{s})$ aflora en el bloque Caborca y la componen las formaciones El Arpa, Caborca, Clemente, Cuarcita Pitiquito, Gamuza, Papalote, Cuarcita Tecolote y La Ciénega de la región de Caborca, y los grupos Las Víboras, El Aguila y Cerro Las Bolas en el centro y oriente del estado (Stewart et al., 2002). En este mapa se distinguen las localidades donde ocurre la transición de la secuencia Neoproterozoica a las formaciones cámbricas $(\boldsymbol{P E}-\boldsymbol{C})$. Las rocas paleozoicas son sedimentarias marinas que van del Cámbrico al Pérmico y se distinguen sucesiones cratónicas y de plataforma $(\boldsymbol{P} z \boldsymbol{p})$ en los bloques Mazatzal y Caborca (Stewart y Poole, 2002), y de cuenca marina profunda (Pzc) en el terreno Cortés (Poole et al., 2005). En estas últimas se incluyen a los pequeños y escasos afloramientos de la Formación Mina México que es depósito sinorogénico de antepaís del alóctono de Sonora (Poole et al., 2005). La unidad Pzm incluye a rocas metasedimentarias y metavolcánicas paleozoicas del Complejo Álamos (Vega-Granillo et al., 2019), que es parte metamorfizada del terreno Cortés, así como a los escasos afloramientos de la Formación Río Fuerte del límite Sinaloa-Sonora (Vega-Granillo et al., 2011).

Otras rocas paleozoicas corresponden a intrusivos graníticos pérmicos que afloran en el noroeste del estado, los cuales junto con granitos triásicos de esa misma región se incluyen en la unidad Pm-Trig. Este conjunto ígneo tiene rango de edad de ca. 275 a $221 \mathrm{Ma}$ (Arvizu et al., 2009; Arvizu e Iriondo, 2015). Los ortogneises triásicos y jurásicos (ca. 250 a $159 \mathrm{Ma}$ ) que afloran en el sur del estado (Sarmiento-Villagrana et al., 2016) dentro del Complejo Sonobari (Mullan, 1978), se cartografían como PzTrJsm.

El Grupo Barranca (Trgb) compuesto por las formaciones Arrayanes, Santa Clara y Coyotes, de edad Triásico-Jurásico(?), representa ambientes continentales, deltáicos y marinos someros en el centro y sur de Sonora, donde sobreyace discordantemente a rocas de los terrenos Caborca y Cortés (Stewart y Roldán-Quintana, 1991). Las rocas del Grupo El Antimonio (Tr) afloran desde el centro al noroeste de Sonora y representan principalmente ambientes marinos (González-León et al., 2005). Dentro de este grupo se cartografía por separado la Formación Sierra de Santa Rosa del Jurásico Temprano (Ji).

También durante el Jurásico se desarrolló el arco volcánico continental Cordillerano (Busby-Spera, 1988) que aflora en la parte norte del estado. Consiste principalmente de riolitas, depósitos volcaniclásticos, cuarzarenitas y conglomerados (Anderson y Silver, 2005; González-León et al., 2020b) que se car- tografían como $\boldsymbol{J v}$ y como $\mathbf{J v m}$, cuando están metamorfizados. El equivalente plutónico $(\boldsymbol{J g})$ de este arco son granitoides, generalmente metamorfizados y con edades de cristalización de ca. 190 a 158 Ma (González-León et al., 2020b). En Jg se incluyen también algunos afloramientos no fechados que han sido interpretados como parte del arco (Anderson y Silver, 2005). Otros afloramientos de rocas metasedimentarias y metavolcánicas de probable edad Triásica y/o Jurásica, que afloran en la región costera de Sonora (Gastil y Krummenacher, 1976), se reconocen como Mzm.

Durante el Jurásico Tardío se desarrollaron las cuencas extensionales Altar-Cucurpe, en la parte central del estado, y la Huachuca, en el noreste de Sonora y sureste de Arizona (Lawton et al., 2020). En la cuenca Altar-Cucurpe se acumularon sedimentos marinos de la Formación Cucurpe (Js) y en la Huachuca se depositó el Conglomerado Glance (Bilodeau et al., 1987) de origen continental. El Conglomerado Glance se cartografía como parte del Grupo Bisbee $(\boldsymbol{K} \boldsymbol{b})$ que está además constituido por las formaciones Cerro de Oro (y su equivalente lateral la Formación Rancho La Colgada), Morita, Caliza Mural, Cintura y La Juana del Cretácico Inferior (resumido en Lawton et al., 2020 y González-León et al., 2020a). En el centro y sur de Sonora aflora el grupo Lampazos $(\boldsymbol{K l})$ que es equivalente lateral de $\boldsymbol{K} \boldsymbol{b}$, pero de facies de cuenca marina más profunda; en $\boldsymbol{K} \boldsymbol{l}$ se incluyen afloramientos indiferenciados de esa edad, del sur de Sonora. A inicios del Cretácico Tardío empezó la fase de acortamiento tectónico Laramide (Valencia-Moreno y Ortega-Rivera, 2011; Calmus et al., 2011) que acumuló los depósitos sintectónicos de la Formación Cocóspera $(\boldsymbol{K} \boldsymbol{f} \boldsymbol{c})$, del Complejo Altar (Jacobson et al., 2019) y el Grupo Cabullona $(\boldsymbol{K} s)$, y simultáneamente empezó la actividad magmática del arco Laramide. Las rocas volcánicas de éste, que se asignan a la Formación Tarahumara $(\boldsymbol{K} \boldsymbol{s}-\boldsymbol{T} \boldsymbol{v})$, tienen edades de cristalización entre ca. 90 y 57 Ma y su plutonismo está representado por abundantes intrusivos $(\boldsymbol{K}-\boldsymbol{T} \boldsymbol{i})$, con edades de cristalización entre ca. 91 y $50 \mathrm{Ma}$ (McDowell et al., 2001; Roldán-Quintana et al., 2009; González-León et al., 2011; González-León et al., 2017a y b). Sin embargo, del centro y sur de Sonora se han fechado cuerpos dioríticos con edades de ca. 95 a $100 \mathrm{Ma}$ (Pacheco Hoyos, 2013; Pérez-Segura et al., 2013; Sarmiento-Villagrana et al., 2016; Vega-Granillo et al., 2019). En este trabajo se intenta reconocer a un tipo de plutones (Tic) leucocráticos, peraluminosos, generalmente de dos micas y con granate de ca. 60 a $32 \mathrm{Ma}$, que ocurren en áreas de complejos de núcleos metamórficos en el norte de Sonora (González-León et al., 2011; González-Becuar et al., 2017; González-León et al. datos no publicados, incluidos en Tabla 1 del Material Suplementario).

Posterior a los eventos laramídicos, en Sonora, ocurrió el volcanismo de la Sierra Madre Occidental (Tvo), que fue seguido por la fase extensional de Sierras y Valles Paralelos que formó horsts y grabens de rumbo casi N-S (resumido en Calmus et al., 2011). En los grabens se acumuló volcanismo félsico y máfico entre ca. 27 y $17 \mathrm{Ma}$, el cual se presenta intercalado con sedimentos aluviales y fluviales de la Formación Báucarit (King, 1939). En este trabajo, y hasta donde es posible, se carto- 
MAPA GEOLÓGICO Y BASE DE DATOS GEOCRONÓLOGICOS DE SONORA, MÉXICO

$114^{\circ} \mathrm{W}$

\section{$112^{\circ} \mathrm{W}$}

$110^{\circ} \mathrm{W}$

Carlos.M. González-León ${ }^{1}$, Gilberto S. Moreno-Hurtado²

${ }^{1}$ Estación Regional del Noroeste, Instituto de Geología, Universidad Nacional Autónoma de México, Hermosillo, México 83000.cmgleon@unam.mx ${ }^{2}$ Universidad Estatal de Sonora, Ingeniería en Geociencias, Hermosillo, México 83140. gilberto.moreno@ues.mx

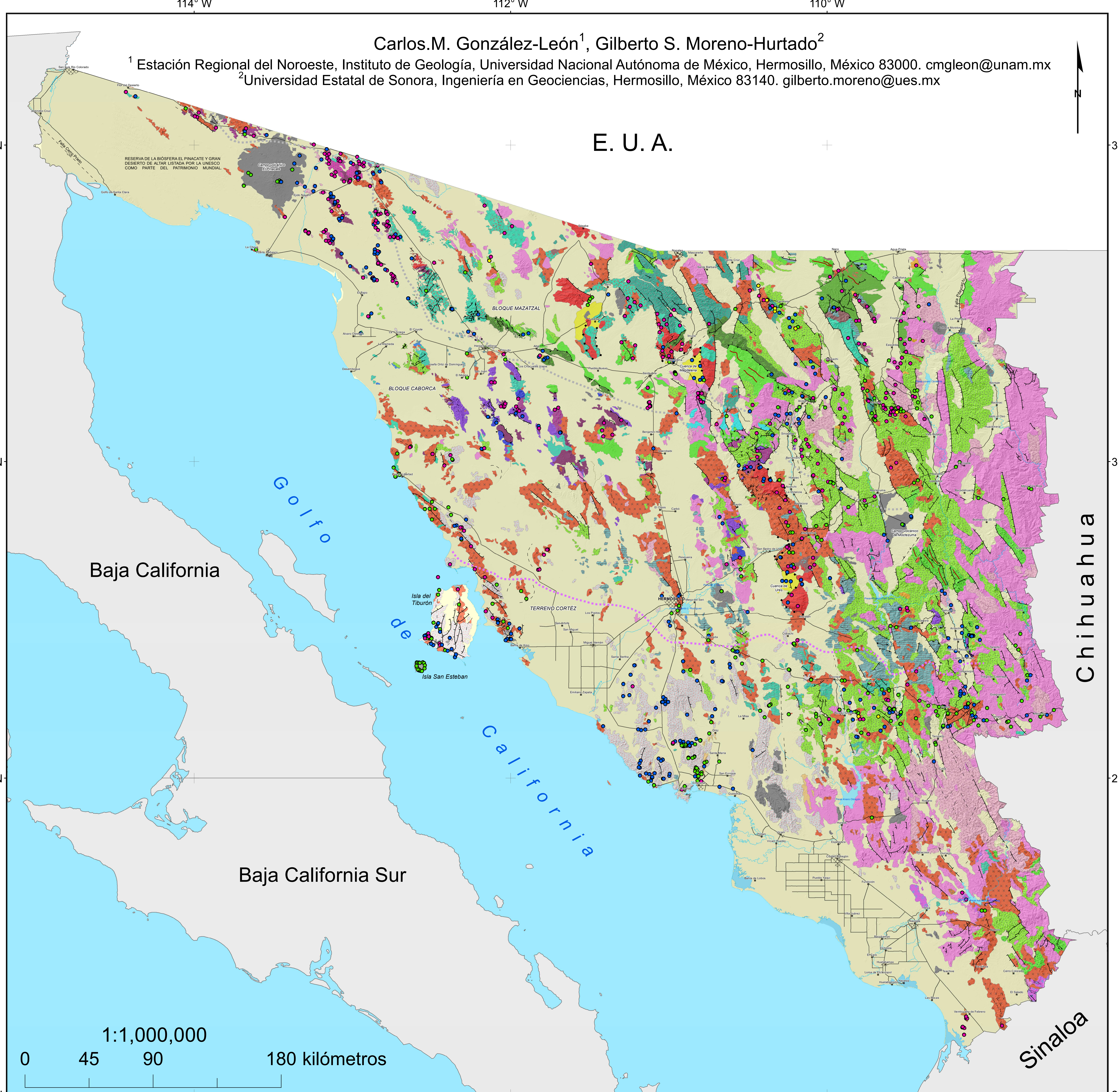

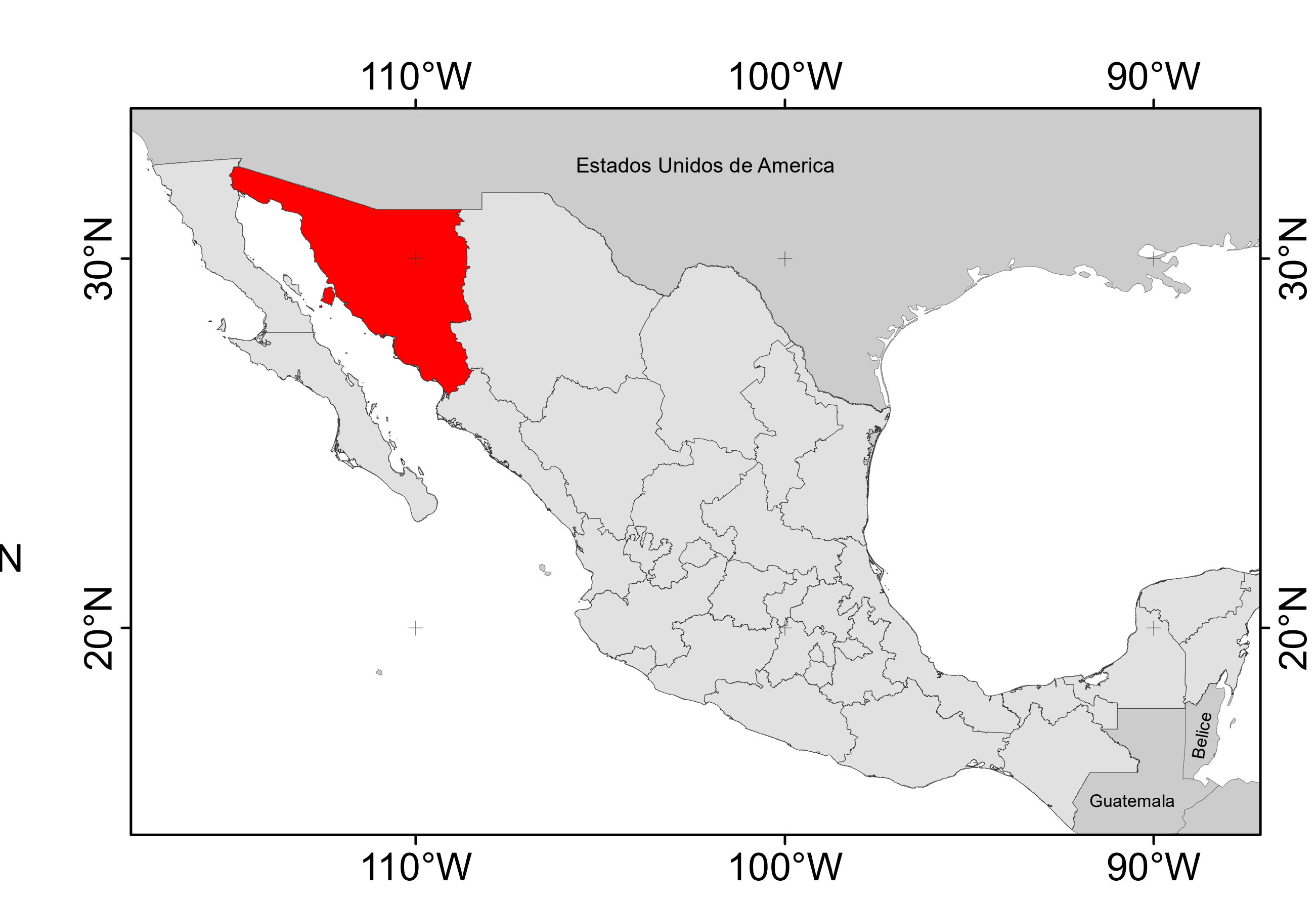

UNIDADES GEOLÓGICAS

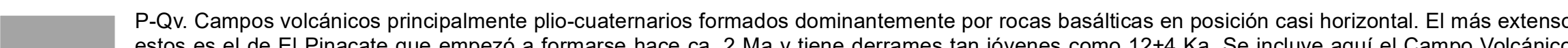
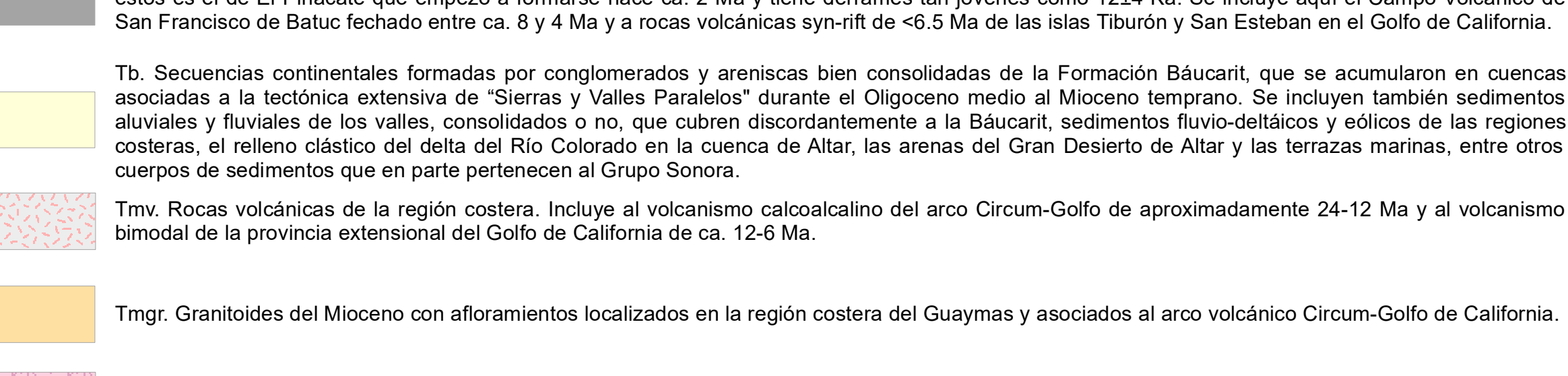

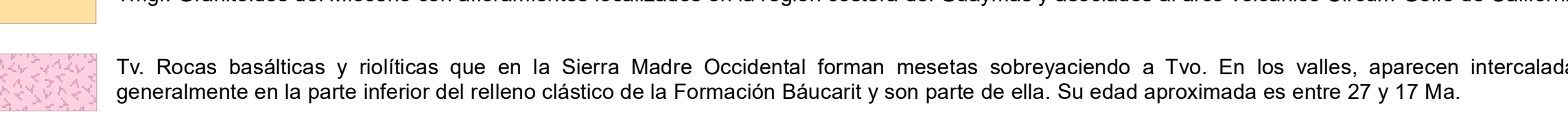

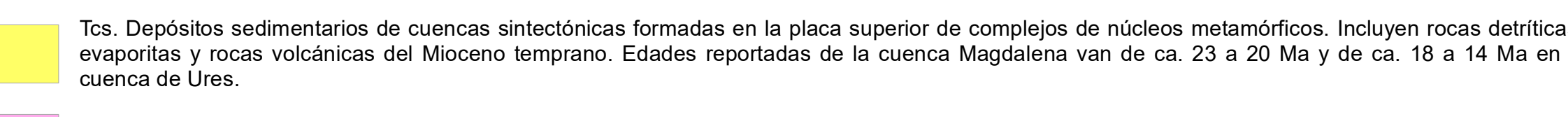

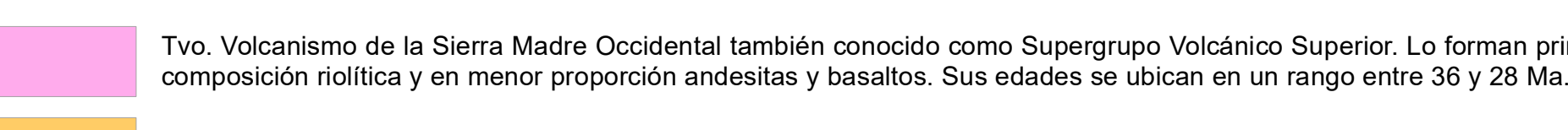

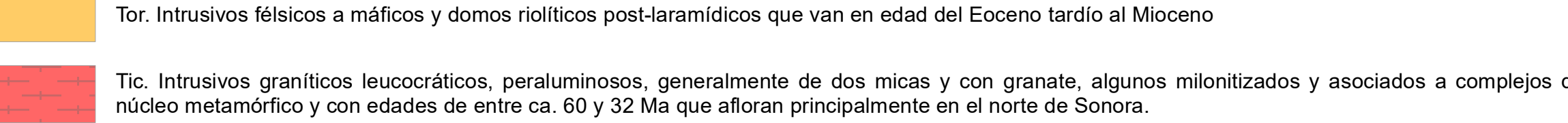

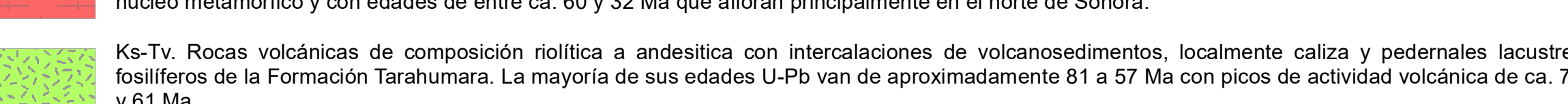

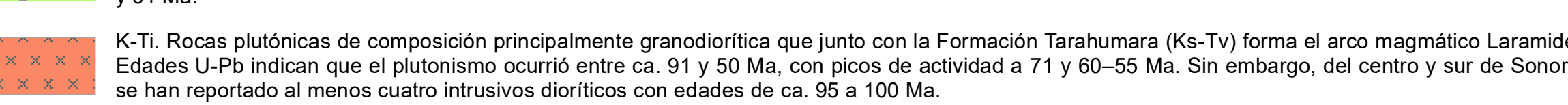

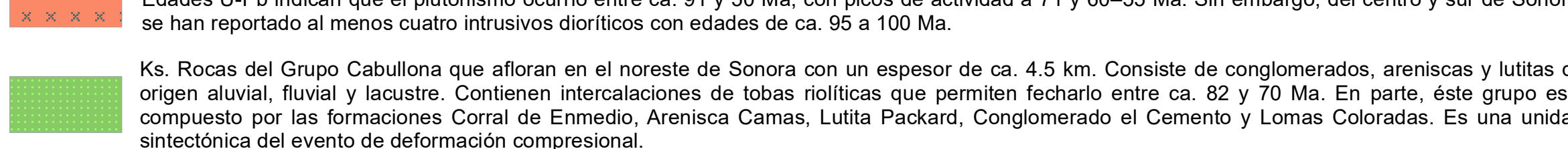

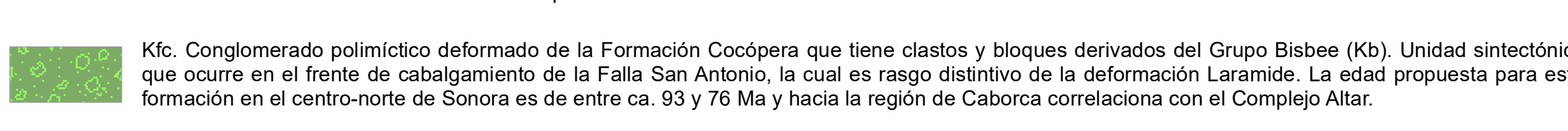

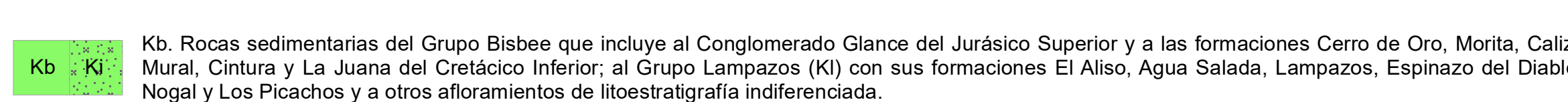

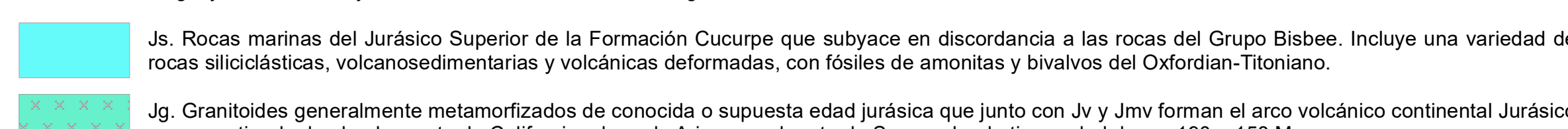

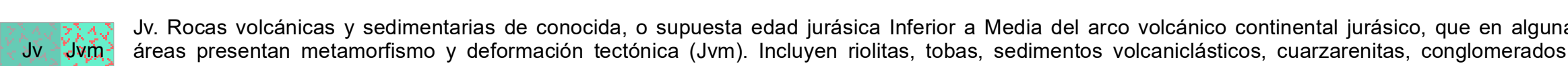

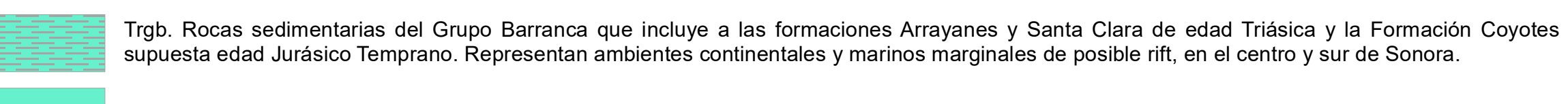

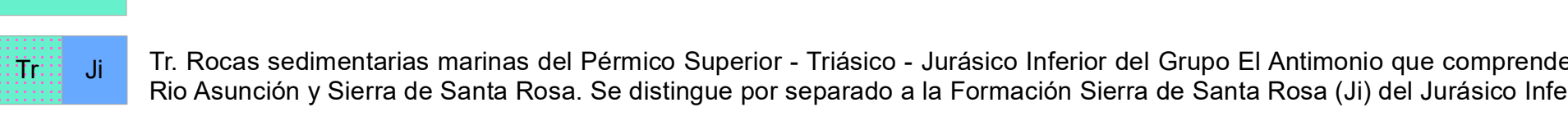

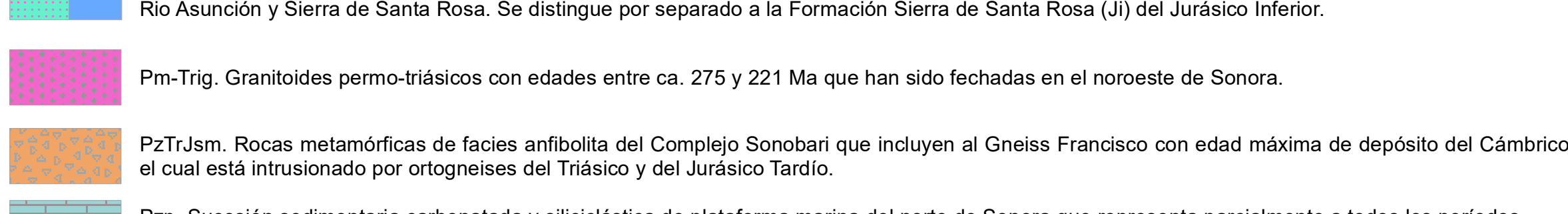

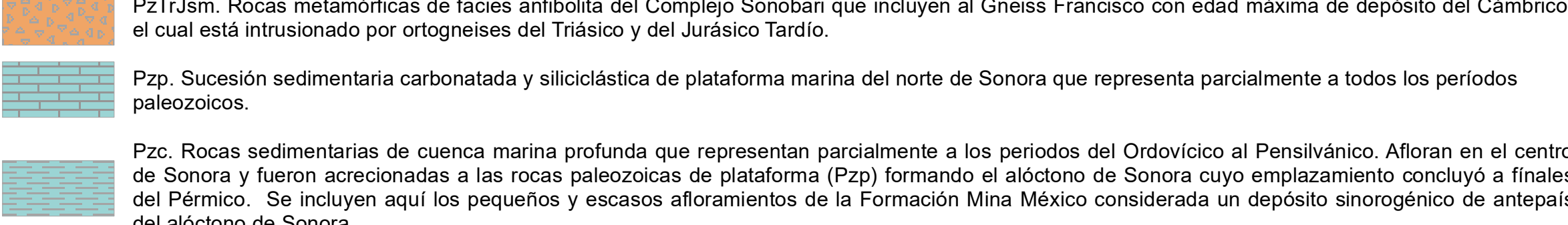

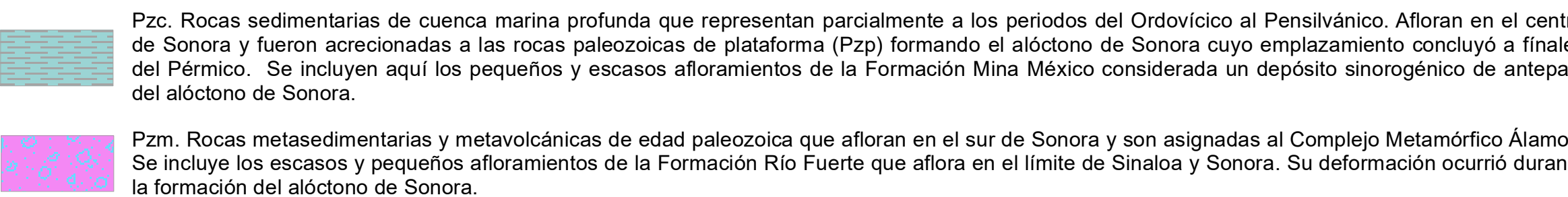

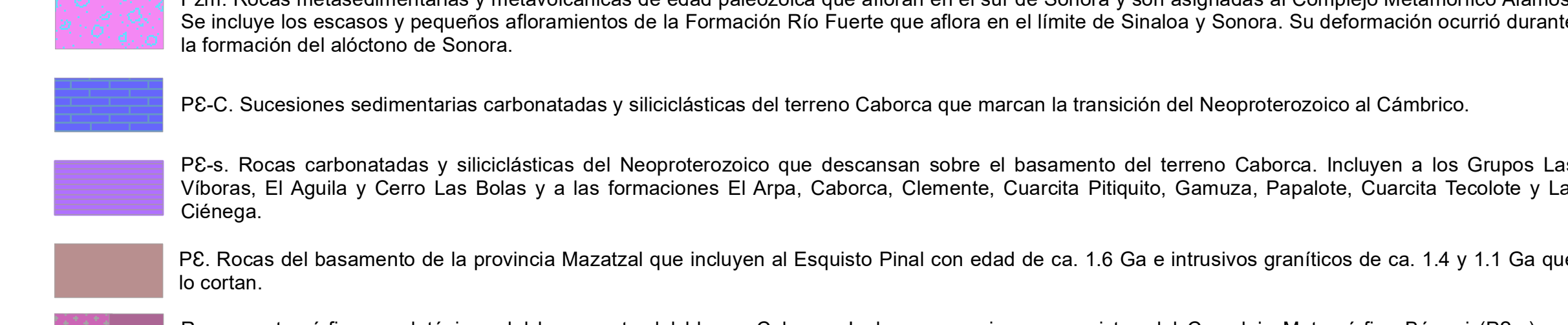

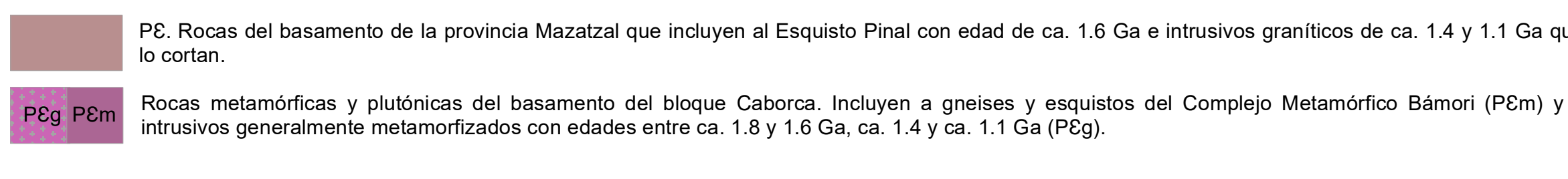

GEOLOGÍA ESTRUCTURAL Traza del frente del oŕgeno Sono
acuerdo a Poole et al. (2005)

GEOCRONOLOGÍA Traza aproximada de la megacizalla Mojave-
Sonora de acuerdo a Anderson y Siver (2005) Método

Falla de Despegue

○ U-Pb

Falla Inversa

Falla Normal

Falla Inferica
Pliegue

Falla de rumbo 
grafían por separado estas rocas volcanicas $(\boldsymbol{T} v)$ que ocurren principalmente en la parte inferior de la Formación Báucarit (incluída en $\boldsymbol{T} \boldsymbol{b}$ ), la cual, hacia su parte superior, está compuestra por rocas silicilásticas continentales. Del mismo modo, se reconoce como una unidad separada (Tor) a los intrusivos félsicos a máficos y a domos riolíticos asociados que han sido fechados con edades entre ca. 36 y 20 Ma. Durante la fase extensiva se formaron complejos de núcleo metamórfico que desarrollaron cuencas sintectónicas con volcanismo bimodal, sedimentos clásticos y evaporíticos (Tcs), como la de Magdalena, que tiene magmatismo de ca. 23 a $20 \mathrm{Ma}$ (Miranda-Gasca y De Jong, 1992).

En $\boldsymbol{T m} \boldsymbol{v}$ se incluye al volcanismo miocénico calcoalcalino de la región costera que pertenece al arco Circum-Golfo y que tiene edad de ca. 24-12 Ma (Mora-Alvarez y McDowell, 2000; Mora-Klepeis y McDowell, 2004), así como al volcanismo postsubducción de la provincia extensional del Golfo de California de ca. 12-6 Ma (Oskin, 2002; Vidal Solano et al., 2007). También se distinguen los escasos granitoides miocénicos (Tmgr) reportados, de la región de Guaymas, y asociados al arco volcánico Circum-Golfo de California (Paz Moreno, 1992; MoraAlvarez y McDowell, 2000).

Dentro de los rellenos de valles $(\boldsymbol{T} \boldsymbol{b})$, se incluyen, además de la Formación Báucarit, a sedimentos más jóvenes, poco o no consolidados, que abarcan los depósitos aluviales y fluviales, sedimentos fluvio-deltáicos y eólicos de regiones costeras, el relleno clástico del delta del Río Colorado (Pacheco et al., 2006), las arenas del Gran Desierto de Altar y las terrazas costeras marinas (Ortlieb, 1991). Estos pertenecen, en parte, al Grupo Sonora de Grijalva-Noriega y Roldán-Quintana (1998). El volcanismo más joven $(\boldsymbol{P}-\boldsymbol{Q v})$ incluye a los campos volcánicos plio-cuaternarios formados dominantemente por rocas basálticas en posición casi horizontal. El más extenso de estos es El Pinacate, que empezó a formarse hace ca. 2 Ma y tiene derrames tan jóvenes como 12,000 \pm 4 años (Gutmann et al., 2000). En $\boldsymbol{P}-\boldsymbol{Q} \boldsymbol{v}$ se incluye también a los campos volcánicos basálticos como el de San Francisco de Batuc (ubicado a $25 \mathrm{~km}$ al oriente de Hermosillo), fechados entre ca. 8 y 4 Ma (Corella Santacruz, 2017), y al de Moctezuma (Paz Moreno et al., 2003), entre otros; así como a rocas volcánicas syn-rift de $<6.5$ Ma de las islas Tiburón (Bennett et al., 2015) y San Esteban (Calmus et al., 2008).

La base de datos geocronológicos (Tabla 1 del Material Suplementario) consultable desde la versión interactiva del mapa (capa GEOCRONOLOGÍA), contiene un total de 1129 edades. Estas corresponden a edades $\mathrm{U}-\mathrm{Pb}$ de rocas ígneas que se consideran de cristalización, y edades $\mathrm{K}-\mathrm{Ar}$ y ${ }^{40} \mathrm{Ar} /{ }^{39} \mathrm{Ar}$ que pueden corresponder a edades de cristalización, en rocas volcánicas, y de enfriamiento o reseteo, en rocas intrusivas (para mayor información sobre esto se recomienda consultar las fuentes originales que se citan en la Tabla 1 del material Suplementario o bien la tabla de atributos de la capa GEOCRONOLOGÍA). También se incluyen edades de mineralización por Re/Os y las edades ${ }^{40} \mathrm{Ar} /{ }^{39} \mathrm{Ar}$ de micas blancas hidrotermales en vetas de cuarzo, que se interpretan como edades de mineralización de los depó- sitos del cinturón de oro orogénico de Caborca (Izaguirre et al., 2017).

\section{Agradecimientos}

González-León agradece a los Drs. Martín A. Valencia Moreno, Benito Noguéz Alcántara y Eduardo Salvatierra Domínguez quienes ayudaron en la elaboración de la versión previa del presente mapa. También agradecemos las valiosas revisiones del Dr. Alexander Iriondo y un árbitro anónimo al presente trabajo, asi como a Estefany Grijalva, Natalia Nevárez e Hiram Morales, estudiantes del programa Ingeniería en Geociencias de la Universidad Estatal de Sonora quienes ayudaron en la compilación cartográfica y geocronológica.

\section{Funding}

González-León agradece a los siguientes proyectos que financiaron cartografía y edades radiométricas aquí reportadas: CONACYT 1494-T9207, CONACYT 24893, CONACYT 253545, PAPIIT-UNAM IN101811-3 y a la Compañía Mexicana de Cobre, S.A. de C.V a través del proyecto institucional "Cartografía geológica, geocronología, petrografía y geoquímica del cuadrángulo La Caridad-Cananea, norte de Sonora".

\section{Referencias}

Aguilera, J. G., 1888. Estudio de los fenómenos séismicos del 3 de mayo de 1887. Anales del Ministerio de Fomento de la República Mexicana 10, 556.

Anderson, T. H., Silver, L. T., 2005. The Mojave-Sonora megashear-Field and analytical studies leading to the conception and evolution of the hypothesis. En: Anderson, T. H., Nourse, J. A., McKee, J. W., Steiner, M. B. (Eds.), The Mojave-Sonora megashear hypothesis: Development, assessment, and alternatives. Vol. 393 of GSA Special Papers. Geological Society of America, pp. 1-50, https://doi.org/10.1130/0-8137-2393-0.1.

Arvizu, H. E., Iriondo, A., 2015. Control temporal y geología del magmatismo Permo-Triásico en Sierra Los Tanques, NW Sonora, México: Evidencia del inicio del arco magmático cordillerano en el SW de Laurencia. Boletín de la Sociedad Geológica Mexicana 67 (3), 545-586, https://dx.doi.org/10.18268/BSGM2015v67n3a16.

Arvizu, H. E., Iriondo, A., Izaguirre, A., Chávez-Cabello, G., Kamenov, G. D., Solís-Pichardo, G., Foster, D. A., Lozano-Santa Cruz, R., 2009. Rocas graníticas pérmicas en la Sierra Pinta, NW de Sonora, México: Magmatismo de subducción asociado al inicio del margen continental activo del SW de Norteamérica. Revista Mexicana de Ciencias Geológicas 26 (3), 709-728.

Bennett, S. E. K., Oskin, M. E., Dorsey, R. J., Iriondo, A., Kunk, M. J., 2015. Stratigraphy and structural development of the southwest Isla Tiburón marine basin: Implications for latest Miocene tectonic opening and flooding of the northern Gulf of California. Geosphere 11 (4), 977-1007, https://doi.org/10.1130/GES01153.1.

Bilodeau, W. L., Kluth, C. F., Vedder, L. K., 1987. Regional stratigraphic, sedimentologic and tectonic relationships of the Glance Conglomerate in southeastern Arizona. En: Dickinson, W. R., Klute, M. A. (Eds.), Mesozoic Rocks of Southern Arizona and Adjacent Areas. Vol. 18. Arizona Geological Society Digest, pp. 229-256.

Busby-Spera, C. J., 1988. Speculative tectonic model for the early Mesozoic arc of the southwest Cordilleran United States. Geology 16 (12), 1121-1125, https://doi.org/10.1130/0091-7613(1988)016<1121:STMFTE>2.3.CO;2. 
Calmus, T., Pallares, C., Maury, R. C., Bellon, H., Pérez-Segura, E., AguillónRobles, A., Carreño, A.-L., Bourgois, J., Cotten, J., Benoit, M., 2008. Petrologic diversity of Plio-Quaternary post-subduction volcanism in northwestern Mexico: An example from Isla San Esteban, Gulf of California. Bulletin de la Société Géologique de France 179 (5), 465-481, https://doi.org/10.2113/gssgfbull.179.5.465.

Calmus, T., Vega-Granillo, R., Lugo-Zazueta, R., 2011. Evolución geológica de Sonora durante el Cretácico Tardío y el Cenozoico. En: Calmus, T. (Ed.), Panorama de la geología de Sonora, México. Vol. 118. Boletín, Instituto de Geología, UNAM, pp. 227-266.

Campa, M. F., Coney, P. J., 1983. Tectono-stratigraphic terranes and mineral resource distributions in Mexico. Canadian Journal of Earth Sciences 20 (6), 1040-1051, https://doi.org/10.1139/e83-094.

Coney, P. J., Campa, M. F., 1987. Lithotectonic terrane map of Mexico (west of the 91st meridian), MF-1874-D, 1:2,500,000 [mapa]. Miscellaneous field studies. U.S. Geological Survey.

Corella Santacruz, C. R., 2017. Petrogénesis del volcanismo alcalino máfico Mioceno tardío-Plioceno del campo basáltico San Francisco de Batuc, Sonora central, México [Tesis de maestría]. Universidad de Sonora, Departamento de Geología.

Dickinson, W. R., Lawton, T. F., 2001. Carboniferous to Cretaceous assembly and fragmentation of Mexico. GSA Bulletin 113 (9), 1142-1160, https://doi.org/10.1130/0016-7606(2001)113<1142:CTCAAF>2.0.CO;2.

Fernández-Aguirre, M. A., Monreal, R., Grijalva-Haro, A. S., 1993. Carta Geológica Sonora, 1:500,000 [mapa]. Dirección General de Fomento Minero y Centro de Estudios Superiores del Estado de Sonora.

Gastil, R. G., Krummenacher, D., 1976. Reconnaissance geologic map of coastal Sonora between Puerto Lobos and Bahía Kino, MC-16, 1:150,000 [mapa]. Geological Society of America Map and Chart Series.

Gastil, R. G., Krummenacher, D., 1977. Reconnaissance geology of coastal Sonora between Puerto Lobos and Bahia Kino. GSA Bulletin 88 (2), 189-198, https://doi.org/10.1130/0016-7606(1977)88<189:RGOCSB > 2.0.CO;2.

Gastil, R. G., Krummenacher, D., Minch, J., 1979. The record of Cenozoic volcanism around the Gulf of California. GSA Bulletin 90 (9), 839-857, https://doi.org/10.1130/0016-7606(1979)90<839:TROCVA>2.0.CO;2.

González-Becuar, E., Pérez-Segura, E., Vega-Granillo, R., Solari, L., GonzálezLeón, C.M., Solé, J., López Martínez, M., 2017. Laramide to Miocene syn-extensional plutonism in the Puerta del Sol area, central Sonora, Mexico. Revista Mexicana de Ciencias Geológicas 34, 45-61, https://doi.org/10.22201/cgeo.20072902e.2017.1.401.

González-León, C. M., Madhavaraju, J., Ramírez Montoya, E., Solari, L. A., Villanueva-Amadoz, U., Monreal, R., Sánchez Medrano, P. A., 2020a. Stratigraphy, detrital zircon geochronology and provenance of the Morita formation (Bisbee Group) in northeastern Sonora, Mexico. Journal of South American Earth Sciences 103, 102761, https://doi.org/10.1016/j.jsames.2020.102761.

González-León, C. M., Solari, L. A., Madhavaraju, J., 2017a. Stratigraphy, geochronology and regional tectonic setting of the Late Cretaceous (ca. 82-70 Ma) Cabullona basin, Sonora, Mexico. Journal of South American Earth Sciences 80, 494-511, https://doi.org/10.1016/j.jsames.2017.09.037.

González-León, C. M., Solari, L. A., Solé, J., Ducea, M. N., Lawton, T. F., Bernal, J. P., Becuar, E. G., Gray, F., Martínez, M. L., Santacruz, R. L., 2011. Stratigraphy, geochronology, and geochemistry of the Laramide magmatic arc in north-central Sonora, Mexico. Geosphere 7 (6), 1392-1418, https://doi.org/10.1130/GES00679.1.

González-León, C. M., Solari, L. A., Valencia-Moreno, M., Heimpel, M. A. R., Solé, J., Becuar, E. G., Santacruz, R. L., Arvizu, O. P., 2017b. Late Cretaceous to early Eocene magmatic evolution of the Laramide arc in the Nacozari quadrangle, northeastern Sonora, Mexico and its regional implications. Ore Geology Reviews 81, 1137-1157, https://doi.org/10.1016/j.oregeorev.2016.07.020.

González-León, C. M., Stanley, G. D., Gehrels, G. E., Centeno-García, E., 2005. New data on the lithostratigraphy, detrital zircon and $\mathrm{Nd}$ isotope provenance, and paleogeographic setting of the El Antimonio Group, Sonora, Mexico. En: Anderson, T. H., Nourse, J. A., McKee, J. W., Steiner, M. B. (Eds.), The Mojave-Sonora megashear hypothesis: Development, assessment, and alternatives. Vol. 393 of GSA Special Papers. Geological Society of America, pp. 259-282, https://doi.org/10.1130/0-8137-2393-0.259.

González-León, C. M., Valencia Moreno, M. A., Salvatierra Domínguez, E.,
2006. Mapa geológico de Sonora, México [mapa]. Digital Geosciences. González-León, C. M., Vázquez-Salazar, M., Navarro, T. S., Solari, L. A., Nourse, J. A., Del Rio-Salas, R., Lozano-Santacruz, R., Arvizu, O. P., Valenzuela Chacón, J. C., 2020b. Geology and geochronology of the Jurassic magmatic arc in the Magdalena quadrangle, north-central Sonora, Mexico. Journal of South American Earth SciencesHttps://doi.org/10.1016/j.jsames.2020.103055.

Grijalva-Noriega, F. J., Roldán-Quintana, J., 1998. An overview of the Cenozoic tectonic and magmatic evolution of Sonora, northwestern Mexico. Revista Mexicana de Ciencias Geológicas 15 (2), 145-156.

Gutmann, J. T., Turrin, B. D., Dohrenwend, J. C., 2000. Basaltic rocks from the Pinacate Volcanic Field yield notably young 40Ar/39Ar ages. Eos Trans. AGU 81 (4), 33-37, https://doi.org/10.1029/00EO00018.

Instituto Nacional de Estadística Geografía e Informática (INEGI), 1990a. Hoja Topográfica La Paz, escala 1:1,000,000 [mapa]. Secretaría de Programación y Presupuesto, INEGI.

Instituto Nacional de Estadística Geografía e Informática (INEGI), 1990b. Hoja Topográfica Tijuana, escala 1:1,000,000 [mapa]. Secretaría de Programación y Presupuesto, INEGI.

Izaguirre, A., Iriondo, A., Kunk, M. J., McAleer, R. J., Atkinson, W. W., Martínez-Torres, L. M., 2017. Tectonic Framework for Late Cretaceous to Eocene Quartz-Gold Vein Mineralization from the Caborca Orogenic Gold Belt in Northwestern Mexico. Economic Geology 112 (6), 1509-1529, https://doi.org/10.5382/econgeo.2017.4519.

Jacobson, C. E., Jacques-Ayala, C., Barth, A. P., Garcia y Barragan, J. C., Pedrick, J. N., Wooden, J. L., 2019. Protolith age of the Altar and Carnero complexes and latest Cretaceous-Miocene deformation in the Caborca-Altar region of northwestern Sonora, Mexico. Revista Mexicana de Ciencias Geológicas 36 (1), 95-109, https://doi.org/10.22201/cgeo.20072902e.2019.1.784.

King, R. E., 1939. Geological reconnaissance in northern Sierra Madre Occidental of Mexico. GSA Bulletin 50 (11), 1625-1722, https://doi.org/10.1130/GSAB-50-1625.

Lawton, T. F., Amato, J. M., Machin, S. E., Gilbert, J. C., Lucas, S. G., 2020. Transition from Late Jurassic rifting to middle Cretaceous dynamic foreland, southwestern U.S. and northwestern Mexico. GSA Bulletin 132 (1112), 2489-2516, https://doi.org/10.1130/B35433.1.

Longoria, J. F., Gonzalez, M. A., Mendoza, J. J., Perez, V. A., 1978. Consideraciones estructurales en el Cuadrangulo Pitiquito-La Primavera, NW de Sonora. Boletín del Departamento de Geología 1, 61-67.

López Ramos, E., 1974. Carta Geológica del Estado de Sonora, 1:5,000,000 [mapa]. Instituto de Geología, UNAM.

McDowell, F. W., Roldán-Quintana, J., Connelly, J. N., 2001. Duration of Late Cretaceous-early Tertiary magmatism in east-central Sonora, Mexico. GSA Bulletin 113 (4), 521-531, https://doi.org/10.1130/00167606(2001)113<0521:DOLCET>2.0.CO;2.

Merriam, R., 1972. Reconnaissance Geologic Map of the Sonoyta Quadrangle, Northwest Sonora, Mexico. GSA Bulletin 83 (11), 3533-3536, https://doi.org/10.1130/0016-7606(1972)83[3533:RGMOTS]2.0.CO;2.

Miranda-Gasca, M., DeJong, K. A., Clark, K. F., Roldán-Quintana, J., Schmidt, R. H., 1992. The Magdalena mid-Tertiary extensional basin. En: Geology and Mineral Resources of Northern Sierra Madre Occidental, Mexico. El Paso Geological Society, Field Conference, Guidebook, pp. 377-384.

Molina-Garza, R. S., Iriondo, A., 2007. The Mojave-Sonora megashear: The hypothesis, the controversy, and the current state of knowledge. En: AlanizÁlvarez, S. A., Nieto-Samaniego, Á. F. (Eds.), Geology of México: Celebrating the Centenary of the Geological Society of México:. Vol. 422 of GSA Special Papers. Geological Society of America, pp. 233-259, https://doi.org/10.1130/2007.2422(07).

Mora-Alvarez, G., McDowell, F. W., 2000. Miocene volcanism during late subduction and early rifting in the Sierra Santa Ursula of western Sonora, Mexico. En: Delgado-Granados H., Aguirre-Díaz, G., Stock, J. M. (Eds.), Cenozoic tectonics and volcanism of Mexico. Vol. 334 of GSA Special Papers. Geological Society of America, pp. 123-141, https://doi.org/10.1130/08137-2334-5.123.

Mora-Klepeis, G., McDowell, F. W., 2004. Late Miocene calc-alkalic volcanism in northwestern Mexico: an expression of rift or subduction-related magmatism? Journal of South American Earth Sciences 17 (4), 297-310, https://doi.org/10.1016/j.jsames.2004.08.001.

Mullan, H. S., 1978. Evolution of part of the Nevadan orogen in northwestern 
Mexico. GSA Bulletin 89 (8), 1175-1188, https://doi.org/10.1130/00167606(1978) $89<1175:$ EOPOTN $>2.0 . C O ; 2$.

Nourse, J. A., Premo, W. R., Iriondo, A., Stahl, E. R., 2005. Contrasting Proterozoic basement complexes near the truncated margin of Laurentia, northwestern Sonora-Arizona international border region. En: Anderson, T. H., Nourse, J. A., McKee, J. W., Steiner, M. B. (Eds.), The MojaveSonora megashear hypothesis: Development, assessment, and alternatives. Vol. 393 of GSA Special Papers. Geological Society of America, pp. 123182, https://doi.org/10.1130/2005.2393(04).

Ortega-Gutiérrez, F., Mitre-Salazar, L.M., Roldán- Quintana, J., ArandaGómez, J., Morán-Zentendo, D., Alaniz-Álvarez, S., Nieto-Samaniego, A., 1992. Carta geológica de la República Mexicana, 1:2,000,000 [mapa]. Consejo de Recursos Minerales de México, Instituto de Geología de la Universidad Nacional Autónoma de México.

Ortlieb, L., 1991. Quaternary shorelines along the northeastern Gulf of California; Geochronological data and neotectonic implications. En: PérezSegura, E., Jácques-Ayala, C. (Eds.), Studies of Sonoran geology. Vol. 254 of GSA Special Papers. Geological Society of America, pp. 95-120, https://doi.org/10.1130/SPE254-p95.

Oskin, M. E., 2002. Tectonic evolution of the Northern Gulf of California, Mexico, deduced from conjugate rifted margins of the upper Delfín basin [Tesis de doctorado]. California Institute of Technology, Pasadena, California.

Pacheco, M., Martín-Barajas, A., Elders, W., Espinosa-Cardeña, J. M., Helenes, J., Segura, A., 2006. Stratigraphy and structure of the Altar basin of NW Sonora: Implications for the history of the Colorado River delta and the Salton trough. Revista mexicana de ciencias geológicas 23 (1), 1-22, publisher: scielomx.

Pacheco Hoyos, J. G., 2013. Reconocimiento de las facies intrusivas en el área de Tonichi, Sonora: petrografía, geocronología y geoquímica de elementos traza [Tesis de licenciatura]. Departamento de Geología, Universidad de Sonora.

Paz Moreno, F. A., 1992. Le volcanisme Mio-Plio-Quaternaire de l'etat du Sonora (nord-ouest du Mexique): evolution spatiale et chronologique; implications petrogenetiques [These Docteur en Sciences]. Université de Droit, d'Economie et des Sciences d'Aix-Marseille.

Paz Moreno, F. A., Demant, A., Cochemé, J.-J., Dostal, J., Montigny, R., 2003. The Quaternary Moctezuma volcanic field: A tholeiitic to alkali basaltic episode in the central Sonoran Basin and Range Province, Mexico. En: Scott E. Johnson, Scott R. Paterson, John M. Fletcher, Gary H. Girty, David L. Kimbrough, Arturo Martín-Barajas (Eds.), GSA Tectonic evolution of northwestern México and the southwestern USA. Vol. 374 of GSA Special Papers. Geological Society of America, pp. 439-455, https://doi.org/10.1130/08137-2374-4.439.

Poole, F. G., Perry, W. J. Jr., Madrid, R. J., Amaya-Martínez, R., 2005. Tectonic synthesis of the Ouachita-Marathon-Sonora orogenic margin of southern Laurentia: Stratigraphic and structural implications for timing of deformational events and plate-tectonic model. En: Anderson, T. H., Nourse, J. A., McKee, J. W., Steiner, M.B. (Eds.), The Mojave-Sonora megashear hypothesis: Development, assessment, and alternatives. Vol. 393 of GSA Special Papers. Geological Society of America, pp. 123-182, https://doi.org/10.1130/2005.2393(04).

Pérez Segura, E., González-Partida, E., Roldán-Quintana, J., 2013. Genetic implications of new $\mathrm{Sr}$ and $\mathrm{Nd}$ isotopic data of the intrusive rocks from the Laramide Arc in Northern Sonora, Mexico. Journal of Iberian Geology 39 (1), 131-146, https://doi.org/10.5209/rev_JIGE.2013.v39.n1.41755.

Roldán-Quintana, J., McDowell, F. W., Delgado-Granados, H., ValenciaMoreno, M., 2009. East-west variations in age, chemical and isotopic composition of the Laramide batholith in southern Sonora, Mexico. Revista Mexicana de Ciencias Geológicas 26 (3), 543-563.

Salas, G. A., 1968. Areal geology and petrology of the igneous rocks, Santa Ana quadrangle, Sonora, Mexico [Tesis de maestría]. The University of Oklahoma.

Sarmiento-Villagrana, A., Vega-Granillo, R., Talavera-Mendoza, O., Vidal-
Solano, J. R., 2016. New age constraints on magmatism and metamorphism of the Western Sonobari Complex and their implications for an earliest Late Cretaceous orogeny on northwestern Mexico. Revista Mexicana de Ciencias Geológicas 33 (2), 170-182, https://doi.org/10.22201/cgeo.20072902e.2016.2.494.

Servicio Geológico Mexicano (SGM), 2008. Carta Geológico-Minera Estado de Sonora. Https://mapserver.sgm.gob.mx/Cartas_Online/geologia/sonora.pdf.

Silver, L. T., Anderson, T. H., 1974. Possible left-lateral early to middle Mesozoic disruption of the southwestern North American craton margin. Geological Society of America Abstracts with Programs 6 (7), 955-956.

Stewart, J. H., Amaya-Martínez, R., Palmer, A. R., 2002. Neoproterozoic and Cambrian strata of Sonora, Mexico. En: Barth, A. (Ed.), Rodinian supercontinent to Laurentian Cordilleran margin. Vol. 365 of GSA Special Papers. Geological Society of America, pp. 5-48, https://doi.org/10.1130/0-81372365-5.5.

Stewart, J. H., Poole, F. G., 2002. Inventory of Neoproterozoic and Paleozoic strata in Sonora, Mexico. Open-File Report 02-97. U.S. Geological Survey.

Stewart, J. H., Roldán-Quintana, J., 1991. Upper Triassic Barranca Group; Nonmarine and shallow-marine rift-basin deposits of northwestern Mexico. En: Pérez-Segura, E., Jácques-Ayala, C. (Eds.), Studies of Sonoran geology. Vol. 254 of GSA Special Papers. Geological Society of America, pp. 19-36, https://doi.org/10.1130/SPE254-p19.

Stewart, J. H., Roldán-Quintana, J., 1994. Map showing late Cenozoic extensional tilt patterns and associated structures in Sonora and adjacent areas, México, MF-2238, 1:1,000,000 [mapa]. Miscellaneous Field Studies. U.S. Geological Survey.

Stone, P., Stevens, C. H., 1988. Pennsylvanian and Early Permian paleogeography of east-central California: Implications for the shape of the continental margin and the timing of continental truncation. Geology 16 (4), 330-333, https://doi.org/10.1130/00917613(1988)016<0330:PAEPPO > 2.3.CO;2.

Suter, M., 2007. The first geologic map of Sonora. Boletín de la Sociedad Geológica Mexicana 59 (1), 1-7, http://dx.doi.org/10.18268/BSGM2007v59n1a1.

Valencia-Moreno, M., Ortega-Rivera, A., 2011. Cretácico Tardío-Eoceno medio en el noroeste de México - evolución del arco magmático continental y su contexto geodinámico (orogenia Laramide). En: Calmus, Thierry (Ed.), Panorama de la geología de Sonora, México. Vol. 118. Boletín, Instituto de Geología, UNAM, pp. 201-226.

Vega-Granillo, R., Bourjac-de Anda, A., Vidal-Solano, J. R., Araux-Sánchez, E., 2019. The Alamos Metamorphic Complex, evidence of late Paleozoic collision between Laurentia and Gondwanan blocks in northwestern Mexico. International Journal of Earth Sciences 108 (3), 1013-1027, https://doi.org/10.1007/s00531-019-01693-y.

Vega-Granillo, R., Salgado-Souto, S., Herrera-Urbina, S., Valencia, V., VidalSolano, J. R., 2011. Metamorphism and deformation in the El Fuerte region: their role in the tectonic evolution of NW Mexico. Revista Mexicana de Ciencias Geologicas 28 (1), 10-23.

Vidal-Solano, J. R., Paz-Moreno, F. A., Demant, A., López-Martínez, M., 2007. Ignimbritas hiperalcalinas del Mioceno medio en Sonora Central: revaluación de la estratigrafía y significado del volcanismo terciario. Revista Mexicana de Ciencias Geológicas 24 (1), 47-67.

Whitmeyer, S. J., Karlstrom, K. E., 2007. Tectonic model for the Proterozoic growth of North America. Geosphere 3 (4), 220-259, https://doi.org/10.1130/GES00055.1.

This article accompanies the following material:

Static map:

Interactive map:

10.22201/igg.25940694e.2021.1.76.182

Suppl. Material:

10.22201/igg.25940694e.2021.1.76.183

10.22201/igg.25940694e.2021.1.76.185

